\title{
231126
}

UCRL-ID-126402

\section{Non-Convex Profile Evolution in Two Dimensions Using Volume of Fluids}

\author{
J. Helmsen \\ P. Colella
}

This is an informal report intended primarily for internal or limited extemal This is an informa and conclusions stated are those of the author and may or may not be those of the Labcratory.

Work performed under the auspices of the U.S. Department of Energy by the Lawrence Livermore National Laboratory under Contract W-7405-ENG-48. 


\section{DISCLAIMER}

This document was prepared as an acccount of work sponsored by an agency of the United States Covernment. Neither the United States Government nor the University of California nor any of their employees, makes any warranty, express or implied, or assumes any legal liability or responsibility for the accuracy, completeness, or usefuliness of any information, apparatus, product, or process disclosed, or represents that its use would not infringe privately own rights. Reference herein to any specific commercial products, process, or service by trade name, trademark, manufacturer, or otherwise, does not necessarily constitute or imply its endorsement, recommendation, or favoring by the United States Government or the University of Californin. The views and opinions of authors expressed herein do not necessarily state or reflect those of the United States Covernment or the University of California, and shall not be used for advertising or product endorsement purposes.

This report has been reproduced directly from the best available copy.

Available to DOE and DOE contractors from the Office of Scientific and Technial Information

P.O. Box 62, Oak Ridge, TN 37831

Prices available from (615) 576-8401, FTS 626-8401

Available to the public from the National Technical Information Service

U.S. Department of Commerce 5285 Port Royal Rd. Springfield, VA 2161 


\title{
Non-Convex Profile Evolution in Two Dimensions Using Volume of Fluids
}

\author{
John Helmsen Phillip Colella *
}

25 January 1997

\begin{abstract}
A new Volume of Fluid (VoF) method is applied to the problem of surface evolution in two dimensions (2D). The VoF technique is applied to problems that are representative of those that arise in semiconductor manufacturing, specifically photolithography and ion-milling. The types of surface motion considered are those whose etch rates vary as a function of both surface position and orientation. Functionality is demonstrated for etch rates that are non-convex in regard to surface orientation. A new method of computing surface curvature using divided differences of the volume fractions is also introduced, and applied to the advancement of surfaces as a vanishing diffusive term.
\end{abstract}

\section{Introduction}

Volume of Fluids methods (VoF) [11] are techniques for representing boundaries between two materials in Computational Fluid Dynamics (CFD) calculations. They are typically used when it is necessary to distinguish between two immiscible fluids. When two fluids cannot possess mixed states, it is often useful to represent them in terms of volume fractions. The volume fraction is defined as the fraction of the volume of a region of space (typically a grid cell) occupied by $F$ luid $d_{1}$ divided by the total volume of the region. Each cell has a volume fraction amount $\left(V_{1}\right)$ between 0 and 1 . (Figure 1) In a two fluid state, the rest of the region is occupied by the other fluid with a corresponding volume fraction $V_{2}$ where $V_{2}=1-V_{1}$. In simulations where more than fluids are present, additional fraction numbers can be employed to represent them: $V_{1}, V_{2}$ and $V_{3}=1-V_{1}-V_{2}$.

\footnotetext{
*Lawrence Berkeley National Laboratory, 1 Cyclotron Rd., Berkeley, CA 94720.
} 


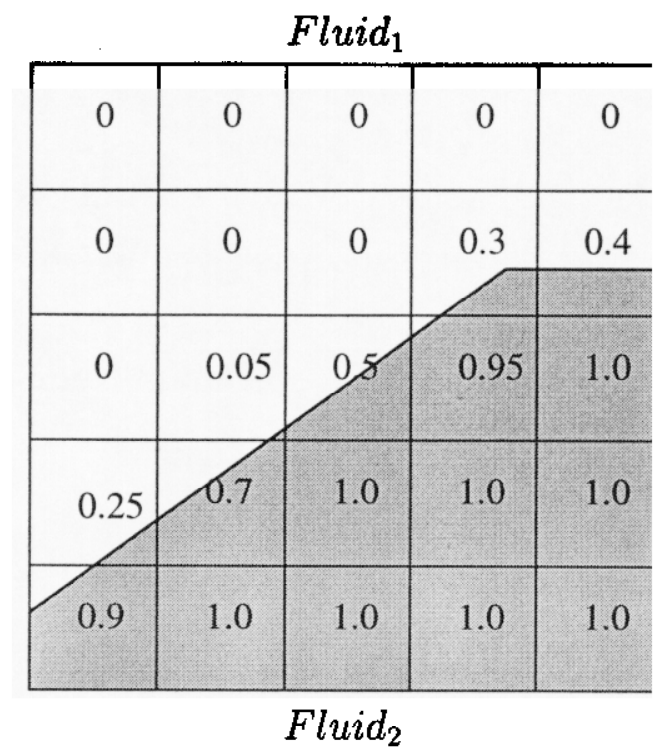

Figure 1: Volume Fractions

Previously VoF techniques were employed for models where the velocity field of the interface was mostly generated by dynamics that occurred off the boundary. These dynamics operated on a time scale similar to the rate of motion of the interface. If the time scale of off boundary dynamics, however, is significantly smaller than the motion of the interface, then these dynamics can be simplified. The interface motion will mostly be determined by the properties of the interface. The rate of motion of the interface, therefore, can be completely derived from the location and orientation of the interface, as in the cases of the semiconductor manufacturing processes [14] of photolithography or unshaded ion milling. This also holds true if the interface dynamics can be sufficiently simplified. Two examples of this simplification, also from semiconductor processing, are plasma etching and chemical vapor deposition, whose off interface dynamics resemble low opacity radiative heat transfer. [15] In this paper, we introduce specially designed computational methods for employing VoF techniques for determining interface motion, when that motion is only a function of the position and the orientation of the interface. Volume of Fluids is then compared to other techniques that have been applied to these problems, such as the string method developed by S. Hamaguchi [5] and the level set method developed by J. Sethian [1][2][9]. 


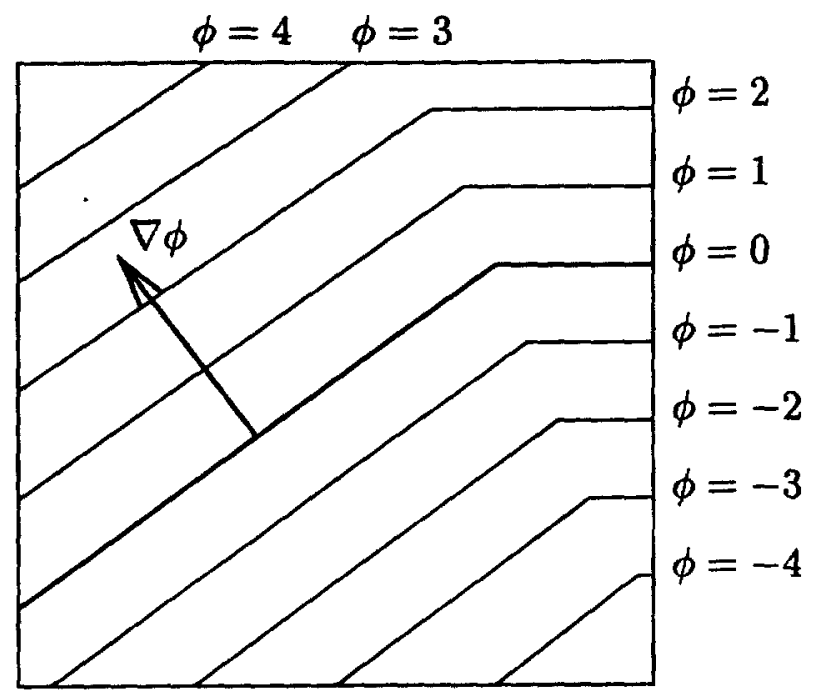

Figure 2: Contours of $\phi$

\section{Surface Motion using Volume of Fluids}

\subsection{The Hamilton-Jacobi Equation}

All first order motion of the interface between two $n$ dimensional regions can be described as special cases of the Hamilton-Jacobi equation:

$$
\phi_{t}+H\left(\phi_{x_{1}}, \phi_{x_{2}}, \ldots \phi_{x_{n}}, x_{1}, x_{2}, \ldots x_{n}, t\right)=0
$$

with certain restrictions on $\phi$. Describing surface evolution in this manner is known as the Level Set approach, and was first described in [9] by S. Osher and J. Sethian. First, the physical location of the interface is represented by $\phi=0$, (Figure 2) or the 0 level-set contour of $\phi$. This contour represents the $n-1$ dimensional surface. The value of $\phi$ is positive inside Fluid $_{1}$ and negative inside Fluid $_{2}$. In addition, $\phi$ is monotonic along paths leaving the surface. $(|\phi|$ increases away from the surface and has no local maxima or minima, although saddle points may be allowed.) $\frac{\nabla \phi}{\|\nabla \phi\|}$ evaluated at $\phi=0$, represents the outward unit surface normal of the region occupied by Fluid, .

$\phi$ is an abstract function that is used only to represent the position of the surface and has no other physical significance. Because the surface is represented only by the 0 level set, the surface properties are gauge invariant 
with respect to $\|\nabla \phi\|$. Therefore, the only valid equations of motion of the surface must be invariant with respect to the gauge. Since the method used to simulate surface advancement will be first order in time, or second order using predictor-corrector techniques, the $t$ dependence in $H$ will be ignored. Therefore, the function can be rewritten as:

$$
\phi_{t}+c\left(\frac{\nabla \phi}{\|\nabla \phi\|}, x_{1}, x_{2}, \ldots, x_{n}\right)\|\nabla \phi\|=0
$$

where all derivatives of $\phi$ are divided by $\|\nabla \phi\|$, in order to maintain $\|\nabla \phi\|$ invariance. In two dimensions, this may be written as:

$$
\phi_{t}+c(\theta, x, y)\|\nabla \phi\|=0
$$

where $\theta=\arctan \frac{\phi_{x}}{\phi_{y}}$. The two dimensional example will be used for the rest of the paper for the purpose of brevity.

Because equation (3) is a first order non-linear equation of the form $F\left(x, y, \phi_{t}, \phi_{x}, \phi_{y}\right)=0$, it can be solved by the method of characteristics. The characteristic curve equations using the curve parameter $t^{\prime}$ are:

$$
\begin{gathered}
\frac{\partial t}{\partial t^{\prime}}=F_{\phi_{t}}=1 \\
\frac{\partial x}{\partial t^{\prime}}=F_{\phi_{x}}=c_{\theta}(\theta, x, y) \cos \theta+c(\theta, x, y) \sin \theta \\
\frac{\partial y}{\partial t^{\prime}}=F_{\phi_{y}}=-c_{\theta}(\theta, x, y) \sin \theta+c(\theta, x, y) \cos \theta \\
\frac{\partial \phi_{x}}{\partial t^{\prime}}=F_{x}=c_{x}(\theta, x, y)\|\nabla \phi\| \\
\frac{\partial \phi_{y}}{\partial t^{\prime}}=F_{y}=c_{y}(\theta, x, y)\|\nabla \phi\| .
\end{gathered}
$$

By combining equations (7) and (8) the characteristic for $\theta$ can also be found.

$$
\frac{\partial \theta}{\partial t^{\prime}}=\frac{\partial \arctan \left(\frac{\phi_{x}}{\phi_{y}}\right)}{\partial t^{\prime}}=c_{x}(\theta, x, y) \cos \theta-c_{y}(\theta, x, y) \sin \theta
$$

By integrating equations (4), (5) and (6) the equations of motion of the surface can be derived:

$$
t=\int \frac{\partial t}{\partial t^{\prime}} d t^{\prime}=\int 1 d t^{\prime}=t^{\prime}+C
$$




$$
\begin{array}{r}
x=\int \frac{\partial x}{\partial t^{\prime}} d t^{\prime}=\int c_{\theta}(\theta, x, y) \cos \theta+c(\theta, x, y) \sin \theta d t^{\prime} \\
=\left(c_{\theta}(\theta, x, y) \cos \theta+c(\theta, x, y) \sin \theta\right) t^{\prime}+C \\
\begin{aligned}
y=\int \frac{\partial y}{\partial t^{\prime}} d t^{\prime} & =\int-c_{\theta}(\theta, x, y) \sin \theta+c(\theta, x, y) \cos \theta d t^{\prime} \\
& =\left(-c_{\theta}(\theta, x, y) \sin \theta+c(\theta, x, y) \cos \theta\right) t^{\prime}+C
\end{aligned}
\end{array}
$$

Therefore, in regions near the surface (i.e. where equation (9) does not significantly contribute) the surface moves according to equations (10), (11) and (12). The constants $C$ are set according to a known position of of the surface at a known time $\left(x_{0}, y_{0}, t_{0}\right)$.

\subsection{The Volume of Fluids Approach}

A moving interface can be solved by the Volume of Fluids approach if the interface is considered to be the boundary of a fluid that is being transported in the characteristic direction of the interface. Flow of volume fractions according to a given velocity field is described by:

$$
f_{t}+\vec{v} \cdot \nabla f=0,
$$

where $f=0$ is the value of $f$ in the section of the plane on the inward side of the curve, and $f=1$ is the value of $f$ on the outward side of the curve. The vector identity $\nabla \cdot(a \vec{b})=a \nabla \cdot \vec{b}+\vec{b} \cdot \nabla a$ generates the equation:

$$
f_{t}+\nabla \cdot(f \vec{v})=f \nabla \cdot \vec{v} .
$$

Equation (14) is in conservation form, with the left hand side representing the conservation terms and the right hand side representing the source term. In the particular application of surface motion, the velocity $\vec{v}$ is given by the characteristics defined in equations (5) and (6):

$$
\vec{v}=\left(c \sin \theta+c_{\theta} \cos \theta\right) \vec{\imath}+\left(c \cos \theta-c_{\theta} \sin \theta\right) \vec{\jmath} .
$$

$\vec{v}$ can also be written as:

$$
\vec{v}=c \vec{n}+c_{\theta} \vec{t}
$$

where $\vec{n}$ is the inward surface normal:

$$
\vec{n}=c \sin \theta \vec{\imath}+c \cos \theta \vec{\jmath}
$$


and $\vec{t}$ is the oriented tangent vector:

$$
\vec{t}=c \cos \theta \vec{\imath}-c \sin \theta \vec{\jmath}
$$

It is important to note that the flow of the fluid contains a tangential component that is not immediately apparent from inspection of equation (3). This tangential flow will greatly assist the generation of stable solutions at corners.

\section{Implementation of Volume of Fluids Approach}

\subsection{Interface Calculation}

To advance the surface, given a collection of volume fractions that define the surface, it is first necessary to determine the orientation of the interface in each cell, since the flow of the fluid is dependent upon the interface orientation. Three conditions of a good interface solver are enforced in the following algorithm. First, the technique must exactly reconstruct the interface if the interface is perfectly linear. Second, if the interface is to be determined at a corner of the interface, it is desirable that a surface normal be found that is between the normals of each edge of the angle. Finally, it is desirable that the method be as fast as possible. For slopes in the range of $45^{\circ}$ to $-45^{\circ}$ (assuming $\Delta x=\Delta y$ ), a $5 \times 3$ stencil vertically aligned is adequate to satisfy all three conditions. Methods employing $3 \times 3$ stencils were employed, but were found to be inadequate as they tended to violate the second and third conditions stated above. Use of a $5 \times 3$ stencil guarantees that the interface will intersect both the left and right hand sides of the stencil, thereby considerably simplifying the problem of interface determination. Because use of a $5 \times 3$ stencil only allows proper determination of the interface in one $90^{\circ}$ section of the plane, the four possible $5 \times 3$ stencils are pre-evaluated to determine which stencil will be used. This is performed by selecting the stencil with the largest total volume fraction in its bottom $2 \times 3$ region, thereby properly orienting the stencil. Therefore, the total stencil for computing the interface for a single cell, is the intersection of two $5 \times 3$ rectangles (Figure 3), one vertical and one horizontal. This stencil is equivalent to a $5 \times 5$ stencil with the corner cells disregarded.

Once the $5 \times 3$ stencil has been determined, (Figure 4) the total volume fractions in each column are determined. The three fraction totals $F_{L}, F_{C}$, and $F_{R}$ are the totals of the left, center and right hand columns respectively. 


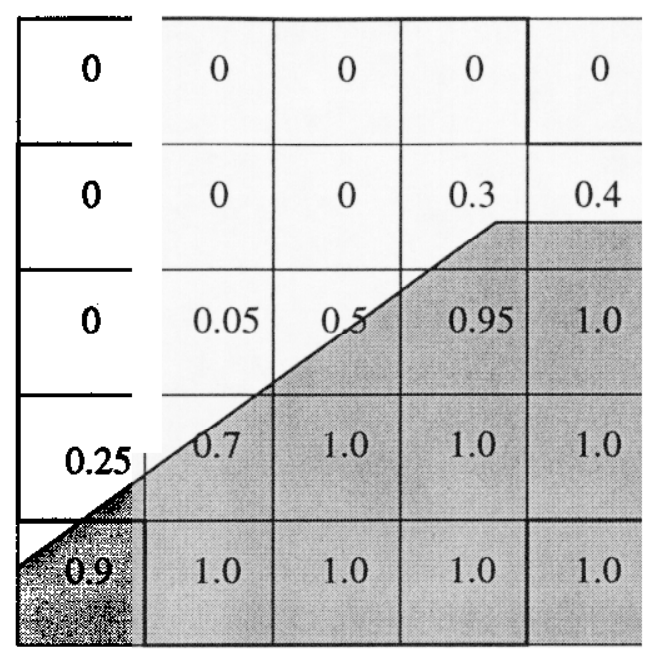

Figure 3: 5x5 Stencil before Rotation

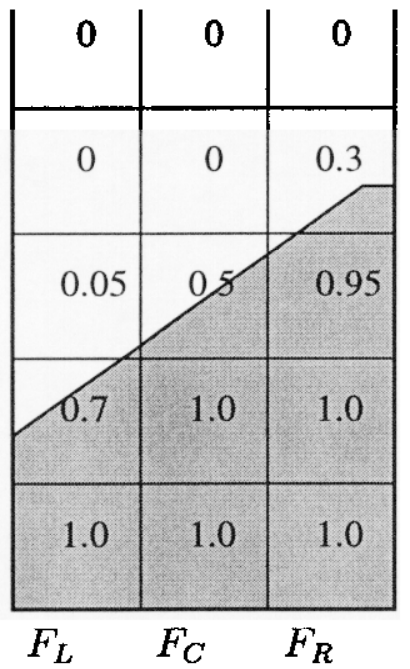

Figure 4: $5 \times 3$ stencil after $0^{\circ}$ Rotation 
The slope of the interface $p$ is determined by the min-mod slope limiting method with centered differencing. First, the terms $S$ and $\Phi$ are determined:

$$
\begin{gathered}
S=\operatorname{sgn}\left(F_{R}-F_{L}\right) \\
\Phi=\left(F_{C}-F_{L}\right)\left(F_{R}-F_{C}\right)
\end{gathered}
$$

If $\Phi<0$ then $p=0$, otherwise:

$$
p=S \min \left(\left|2\left(F_{R}-F_{C}\right)\right|,\left|2\left(F_{C}-F_{L}\right)\right|,\left|0.5\left(F_{R}-F_{L}\right)\right|\right) \frac{\Delta y}{\Delta x}
$$

Once $p$ is determined, it is easily converted either to a unit surface normal or an angle $\theta$. The interface line segment inside the cell is determined by using $p$ as the slope for the line segment. An offset for the line is chosen, so that the area of the intersection of the half plane determined by the line and the boundaries of the cell yield a volume fraction equivalent to the original volume fraction in the cell. After all cells with volume fractions greater than 0 and less than 1 have had their interfaces determined, then the interface will be represented by a collection of line segments that are not necessarily connected at the endpoints.

\subsection{Surface Curvature Calculation}

Fortunately, surface curvature can be determined concurrently with the calculation of the interface slope. Given a function $y=u(x)$ representing the interface, the surface curvature [12] of this line $K$ is known to be:

$$
K=\frac{-u_{x x}}{\left(1+u_{x}^{2}\right)^{\frac{3}{2}}}
$$

This can be computed after the determination of the slope of the interface $p=u_{x}$ in equation (21) using the totals of the volume fractions in the columns $F_{L}, F_{C}$ and $F_{R}$.

$$
K=\frac{2 F_{C}-F_{L}-F_{R}}{\left(1+p^{2}\right)^{\frac{3}{2}}(\Delta x)^{2}} \Delta y
$$

This calculation of the surface curvature gives a cell centered value for the curvature. Because the surface velocities are edge centered values, a curvature at the edges of the interface cells must be determined. This is performed by averaging the curvature values at each edge, if the edge is between two 
interface cells, or by assigning the center value to the edge, if the edge borders only one interface cell. Addition of diffusive curvature to the Volume of Fluids method is added by subtracting $\epsilon K$ from the etch rate function, where $\epsilon$ is a constant proportional to $\Delta x$.

\subsection{Edge Velocity Calculation}

Because the direction of the surface normal $(\theta)$ is constant across each cell and is discontinuous at cell boundaries the source term in equation (14) can be split into a part that varies with position and is constant in $\theta$ and one that varies with $\theta$ and is constant in position. Expanding $\vec{v}$ out, the source term is:

$$
f \nabla \cdot\left(c \vec{n}+c_{\theta} \vec{t}\right)
$$

which becomes via vector identities:

$$
f\left(\nabla c \cdot \vec{n}+c \nabla \cdot \vec{n}+\nabla c_{\theta} \cdot \vec{t}+c_{\theta} \nabla \cdot \vec{t}\right)
$$

where $f\left(\nabla c \cdot \vec{n}+\nabla c_{\theta} \cdot \vec{t}\right)$ is cell centered and $f\left(c \nabla \cdot \vec{n}+c_{\theta} \nabla \cdot \vec{t}\right)$ is edge centered. The cell centered term can be discretized (in two dimensions) as:

$$
\begin{aligned}
& f_{i, j}\left(\left(c_{i+\frac{1}{2}, j}\left(\theta_{i, j}\right) \vec{n}_{x i, j}-c_{i-\frac{1}{2}, j}\left(\theta_{i, j}\right) \vec{n}_{x i, j}\right.\right. \\
& \left.+c_{\theta i+\frac{1}{2}, j}\left(\theta_{i, j}\right) \vec{t}_{x i, j}-c_{\theta i-\frac{1}{2}, j}\left(\theta_{i, j}\right) \vec{t}_{x i, j}\right) / \Delta x \\
& +\left(c_{i, j+\frac{1}{2}}\left(\theta_{i, j}\right) \vec{n}_{y i, j}-c_{i, j-\frac{1}{2}}\left(\theta_{i, j}\right) \vec{n}_{y i, j}\right. \\
& \left.\left.+c_{\theta i, j+\frac{1}{2}}\left(\theta_{i, j}\right) \vec{t}_{y i, j}-c_{\theta i, j-\frac{1}{2}}\left(\theta_{i, j}\right) \vec{t}_{y i, j}\right) / \Delta y\right)
\end{aligned}
$$

where $c_{i+\frac{1}{2}}\left(\theta_{i}\right) \vec{n}_{x i}$ is the value of the etch rate computed using the right hand position of the cell with the cell centered value for $\theta$ multiplied by the $\mathbf{x}$ component of the surface normal vector. The other terms are similar, but use the left hand side of the cell, the tangent vector or the $y$ component. On an edge, the $x$ edge in this example, the edge centered term is discretized as:

$$
\begin{array}{r}
f_{i+\frac{1}{2}, j}\left(c_{i+\frac{1}{2}, j}\left(\theta_{i+1, j}\right) \vec{n}_{x i+1, j}-c_{i+\frac{1}{2}, j}\left(\theta_{i, j}\right) \vec{n}_{x i, j}+\right. \\
\left.c_{\theta i+\frac{1}{2}, j}\left(\theta_{i+1, j}\right) \vec{t}_{x i+1, j}-c_{\theta i+\frac{1}{2}, j}\left(\theta_{i, j}\right) \vec{t}_{x i, j}\right)
\end{array}
$$

The definition of $f_{i+\frac{1}{2}, j}$ is, unfortunately, not directly defined as a result of the mathematical derivation. Therefore, a suitable value is defined through 
the use of geometrical insight. If the condition

$$
\begin{gathered}
c_{i+\frac{1}{2}, j}\left(\theta_{i+1, j}\right) \vec{n}_{x i+1, j}-c_{i+\frac{1}{2}, j}\left(\theta_{i, j}\right) \vec{n}_{x i, j}+ \\
c_{\theta i+\frac{1}{2}, j}\left(\theta_{i+1, j}\right) \vec{t}_{x i+1, j}-c_{\theta i+\frac{1}{2}, j}\left(\theta_{i, j}\right) \vec{t}_{x i, j}<0
\end{gathered}
$$

holds, this represents, in the convex case, the continued propagation of the discontinuity in $\theta$. The removal of material is handled in a geometric manner, which implicitly defines $f_{i+\frac{1}{2}, j}$ as being the appropriate value to match the amount of material that is removed geometrically. The condition:

$$
\begin{gathered}
c_{i+\frac{1}{2}, j}\left(\theta_{i+1, j}\right) \vec{n}_{x i+1, j}-c_{i+\frac{1}{2}, j}\left(\theta_{i, j}\right) \vec{n}_{x i, j}+ \\
c_{\theta i+\frac{1}{2}, j}\left(\theta_{i+1, j}\right) \vec{t}_{x i+1, j}-c_{\theta i+\frac{1}{2}, j}\left(\theta_{i, j}\right) \vec{t}_{x i, j}>0
\end{gathered}
$$

corresponds to the generation of a rarefaction fan at the edge. New material must be introduced. Because maintaining the entropy condition is equivalent to maintaining a slightly rounded corner, $f_{i+\frac{1}{2}, j}$ is chosen as the lowest of $f_{i, j}$ and $f_{i+1, j}$ if the curvature $K>0$, and as the largest of $f_{i, j}$ and $f_{i+1, j}$ if the curvature $K<0$. This maximal or minimal setting of $f_{i+\frac{1}{2}, j}$ acts as a small curvature term added to the advancement rate, thereby helping to maintain the entropy condition.

To determine the speed and direction of shocks, and to accurately compute the behavior of the front in cases of non-convex flow, the theory of conservation laws is applied. The particular method of determining the solution of the non-convex case that is employed here is mostly an application of work done by Hamaguchi [5]. If the shock proceeds from a corner formed by line segments with angles $\theta_{l}$ and $\theta_{r}$, where $\theta=0$ represents a surface whose outward normal points in the positive $y$ direction, and $\theta=\frac{\pi}{2}$ points along the positive $x$ direction. The shock that is formed between them proceeds along a parametric line $(X(t), Y(t))$, where:

$$
\begin{aligned}
& \frac{d X(t)}{d t}=\frac{\cos \theta_{r} c_{l}-\cos \theta_{l} c_{r}}{\sin \left(\theta_{l}-\theta_{r}\right)}, \\
& \frac{d Y(t)}{d t}=\frac{\sin \theta_{l} c_{r}-\sin \theta_{r} c_{l}}{\sin \left(\theta_{l}-\theta_{r}\right)}
\end{aligned}
$$

where $c_{l}=c\left(\theta_{l}, x, y\right)$ and $c_{r}=c\left(\theta_{r}, x, y\right)$.

In the case of non-convex flow, it is possible to have combinations of alternating shocks and rarefaction fans emerging from a single corner. Because rarefaction fans emerging from a corner all take on the same value 
for $f_{i+\frac{1}{2}, j}$, all fans, and shocks between fans, can be considered as one large rarefaction fan. Therefore, the only possibilities of behavior at edges are 1) a single shock, 2) a single fan, 3) a fan bounded on one side by a shock, 4) a fan bounded on two sides by a shock. In order to determine which case arises, it is necessary to examine a selection of the values of $\theta$ between $\theta_{l}$ and $\theta_{r}$.

A user assigned refinement parameter $\Delta \theta_{\max }$ is used to determine the refinement $\Delta \theta$ between $\theta_{l}$ and $\theta_{r} . \Delta \theta$ is first set to $\left\|\theta_{r}-\theta_{l}\right\|$, and is multiplied by $\frac{1}{2}$ until $\Delta \theta<\Delta \theta_{\max }$. The values of $\theta_{i}$ that are evaluated as (assuming $\theta_{l}<\theta_{r}$ ). Once these angles are set, the Hamaguchi method is used to determine the condition of the characteristics that are generated at the discontinuity. Once these characteristics are determined, the horizontal velocities of both the left and rightmost sides of the shock/rarefaction fan generated by the discontinuity are returned to the method. These velocities are designated as shockl and shock.

If shock $_{l}=$ shock $_{r}$ then a single shock has been generated. If $s h o c k_{l}<v_{l}$, where $v_{l}$ and $v_{r}$ are the horizontal velocity components of $c_{i+\frac{1}{2}, j}\left(\theta_{i, j}\right) \vec{n}_{x i, j}+$ $c_{\theta i+\frac{1}{2}, j}\left(\theta_{i, j}\right) \vec{t}_{x i, j}$ and $c_{i+\frac{1}{2}, j}\left(\theta_{i+1, j}\right) \vec{n}_{x i+1, j}+c_{\theta i+\frac{1}{2}, j}\left(\theta_{i+1, j}\right) \vec{t}_{x i+1, j}$ respectively, then material is entering a shock from the left hand side. If $s h o c k_{r}>v_{r}$, then material is entering a shock from the right hand side. If $s$ ock $_{l}<$ shock $_{r}$, then material is being generated in the rarefaction fan. It is possible for all three of the first conditions to hold simultaneously, as well as all three of the last conditions.

\subsection{Flux Calculation}

To determine the amount of material that is flowing across the edges, and to determine the amount that is being created or destroyed according to the source term. Geometrical methods are employed. Given the parameters shock $_{l}$, shock $r, v_{l}, v_{r}, f_{i+\frac{1}{2}, j}$ and the geometrical representations of the surface in each neighboring cell, the operations necessary for the method may be specified. The first major decision point is based on the signs of shock $_{l}$ and shock $_{r}$. Three possibilities exist. In the case of $s$ ock $k_{l}<0$ and shock $_{\tau}<0$ (Figure 7), the entire fan and shock structure penetrates into the left hand cell. Second, for shockl $_{l}>0$ and shock $r>0$ (Figure 6), the fan and shock structure penetrates into the right hand cell. Third, if shockl $<0$ and shock $_{r}>0$, (Figure 5) then the shock and fan structure is split in two through the fan. For the above cases, a smooth transition from one case to 


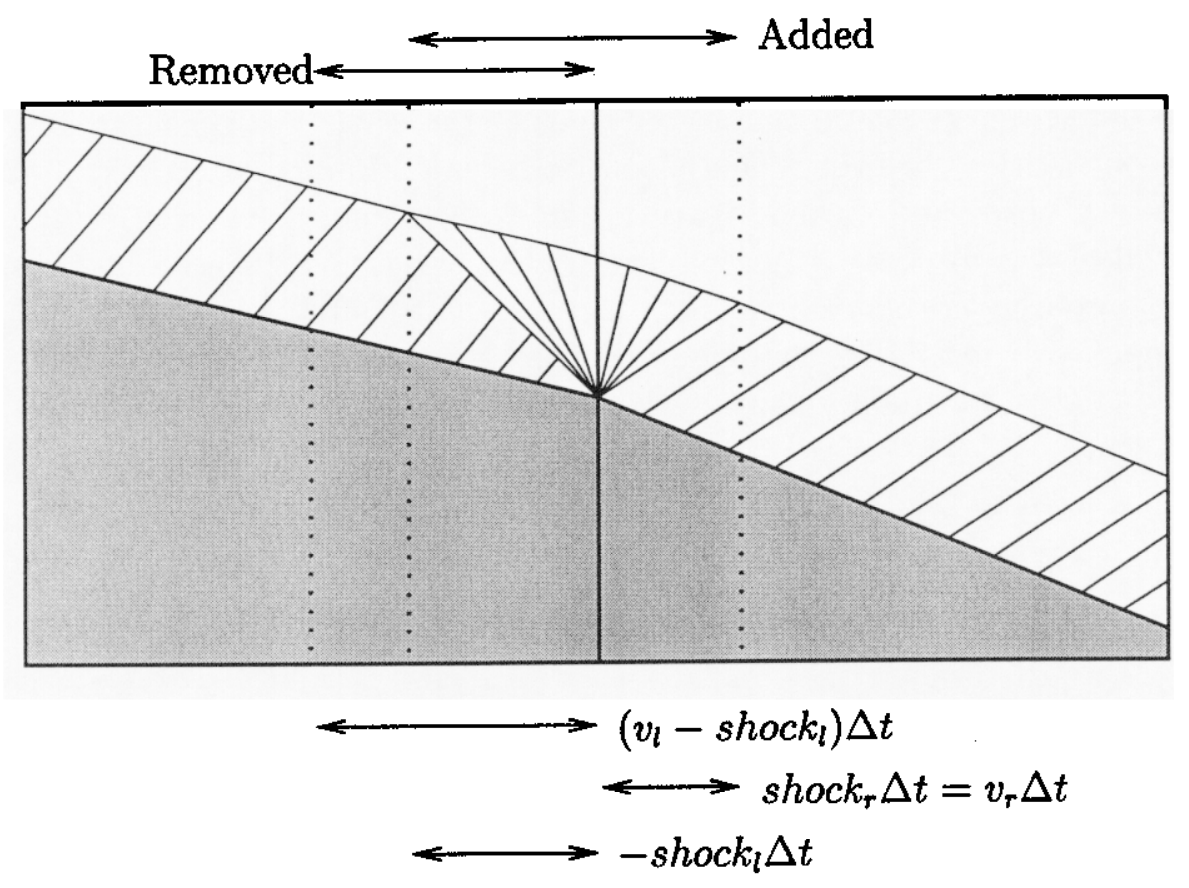

Figure 5: Rarefaction Fan where shock $_{l}<0$ and $s h o c k_{r}>0$ 


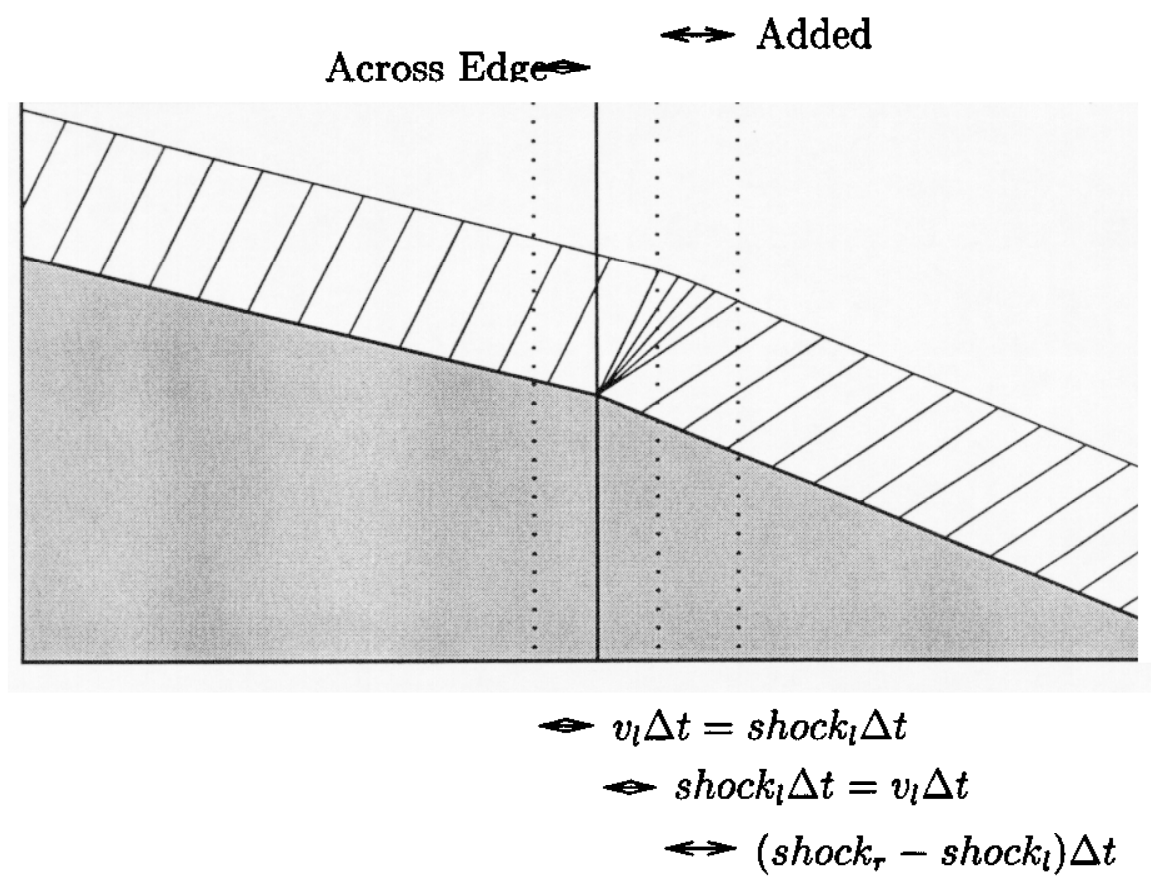

Figure 6: Rarefaction Fan and Shock where $s h o c k_{l}>0$ and $s h o c k_{r}>0$ 


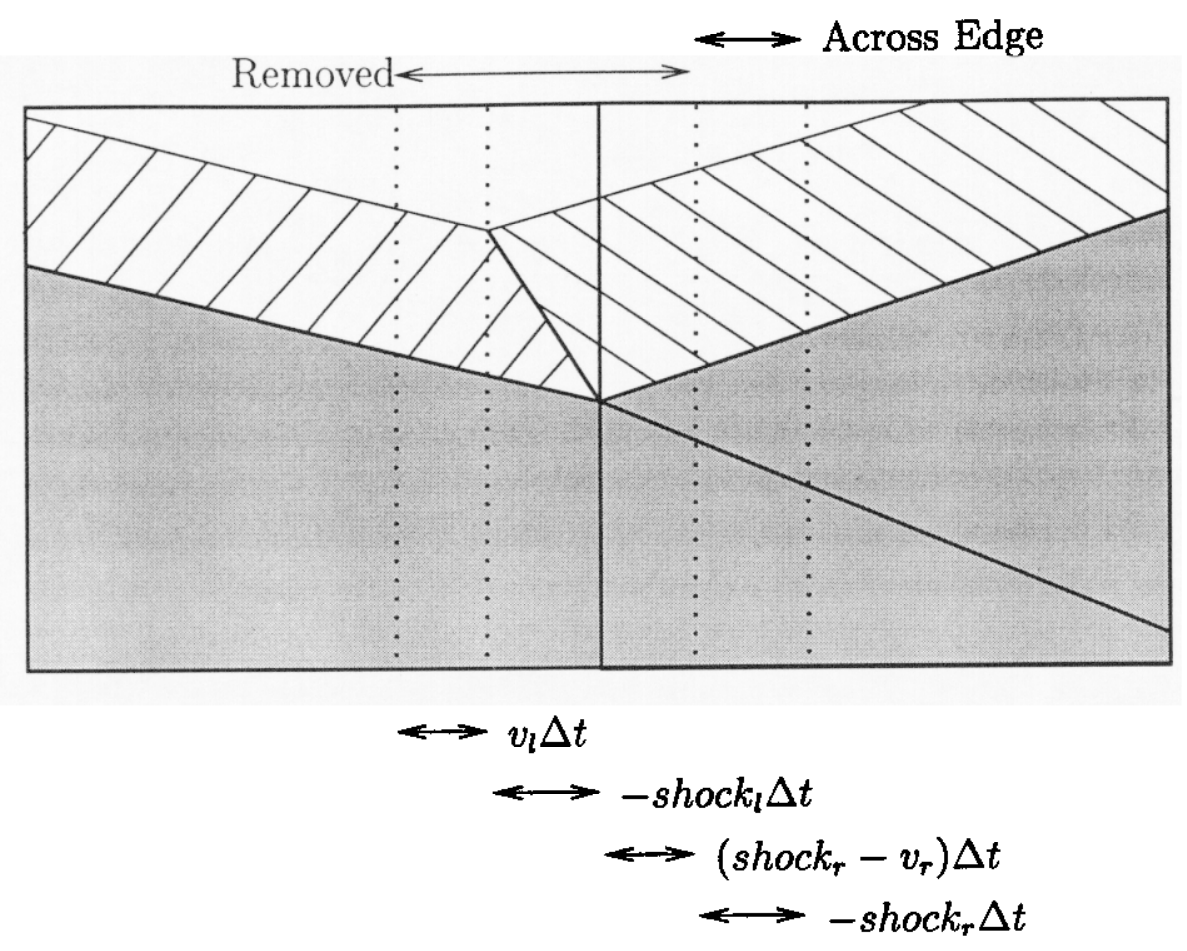

Figure 7: Shock where $\operatorname{shock}_{l}<0$ and $\operatorname{shock}_{r}<0$

another exists through $s h o c k_{l}=0$ and $s h o c k_{r}=0$, so these special cases will not be considered explicitly.

If both shock and $_{\text {shock }}$ have the same sign and are $\neq 0$, then there is transport of material across the edge (Figures $6 \& 7$ ). In this case, the amount of material enclosed by a rectangle that abuts the edge is removed from one cell and added to the other to represent the flow. The rectangle is located in the upwind cell. The height of the rectangle is the height of the edge, and the width of the rectangle is $v_{r} \Delta t$ if the right hand cell is upwind or $v_{l} \Delta t$ otherwise.

If a shock exists in the simulation (i.e., $v_{l} \neq$ shock $_{l}$ or $v_{r} \neq$ shock $_{r}$ ), then the material on both sides of the shock or shocks is removed (Figures $6 \&$ 7). The amount removed is the amount contained in a rectangle that borders the edge with the height of the edge and a width of $\left(v_{l}-s h o c k_{l}\right) \Delta t$ if $v_{l} \neq$ shock $_{l}$ (Figure 6)or a width of $\left(s h o c k_{r}-v_{r}\right) \Delta t$ if $v_{r} \neq s h o c k_{r}$ (Figures 6 $\&$ 7). The rectangle is in the left hand cell for the $s_{h o c k}$ case and the right 
hand cell in the shock $k_{r}$ case. If shock $_{1}>0$, then the material is subtracted from the right hand cell, otherwise it is subtracted from the left hand cell.

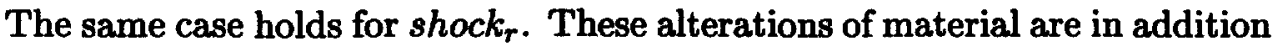
to the alterations above that represent flow across the edge.

If shock $_{l}<$ shock $_{r}$ then a rarefaction fan has occurred (Figures $5 \& 6$ ). If $s_{\text {hock }}<0$ and $s h o c k_{r}<0$, then material of an amount $f_{i+\frac{1}{2}, j}\left(\operatorname{shock}_{r}-\right.$ shock $) \Delta t \Delta y$ is added to the left cell, assuming that the two cells are adjacent in the $\mathrm{x}$-coordinate direction. If shock $_{\mathrm{l}}>0$ and shock $_{r}>0$ (Figure 6), then the same amount is added, but to the right cell. If $s h o c k_{l}<0$ and shock $k_{r}>0$ (Figure 5), then $f_{i+\frac{1}{2}, j}\left(\right.$ shock $\left._{r}\right) \Delta t \Delta y$ is added to the right cell and $f_{i+\frac{1}{2}, j}\left(-s h o c k_{l}\right) \Delta t \Delta y$ is added to the left cell. It is not possible for the case of shock $_{l}>$ shock $_{r}$ to occur.

\subsection{Divergence Calculation}

Now that the edge flux and edge centered divergence term has been accounted for, it is necessary to determine the cell centered divergence term. There are two methods for computing the divergence term, implicit and explicit. Generally, sweep methods in two dimensions alternate between implicit and explicit methods in the pattern: $x$-implicit, y-explicit, $y$-implicit, x-explicit. This alternation generates $O\left(h^{2}\right)$ accuracy under some conditions. However, since the edge centered divergence calculation is performed in an explicit manner for all coordinate sweeps, only $O(h)$ accuracy can be obtained without using a significantly more complicated predictor-corrector method for the edge centered divergence term.

The values $f_{i, j}$ represent the volume fractions at the beginning of the entire advancement procedure. The values $\tilde{f_{i, j}}$ represent the volume fractions after the application of the edge flux and the edge divergence terms are applied to $f_{i, j}$. The values $\overline{f_{i, j}}$ will be the final volume fractions at the end of the advancement procedure.

To compute the cell centered divergence terms implicitly, assuming the $\mathrm{x}$-sweep is being employed, the following equation is used.

$$
\tilde{f_{i, j}}=\frac{\tilde{f_{i, j}}}{1-\frac{\Delta t}{\Delta x}\left(v_{l i+1, j}-v_{r i, j}\right)}
$$

To compute the cell centered divergence term explicitly:

$$
\tilde{f_{i, j}}=\tilde{f_{i, j}}+f_{i, j} \frac{\Delta t}{\Delta x}\left(v_{l i+1, j}-v_{r i, j}\right)
$$




\section{Results}

Four different rate functions were used to test the operation of the algorithm. The functions are listed in order of increasing complexity and represent different types of mathematical models encountered in semiconductor profile simulation. Each of the simulations occurs in a $10 \times 10$ unit space, with the $(0,0)$ point in the upper left. The $x$ direction is horizontal, and $y$ is represented as the vertical. The numbers given in the captions represent the number of cells employed in the simulation along the $\mathrm{x}$ and the $\mathrm{y}$ directions.

\subsection{Lithography}

Lithography is a process that entails the dissolution of a energy sensitive compound. This process is employed to transfer a pattern from either an image mask or the path of a directed beam to the surface of the chip. Exposure of the resist material to an energy source alters the etch rate of the material to a post-exposure developing solution, thereby exposing selected sections of the surface. Although, there are many lithography processes (i.e. e-beam, optical, $\mathrm{X}$-ray, ion-beam), each generates a resist whose dissolution can be described purely in terms of the etch rate as a function of position $[3][4][6][8][13]$.

In this example, the etch rate function is:

$$
R(x, y)=e^{-0.32(x-5)^{2}}\left(\left(\cos \left(0.4 y \pi+\frac{\pi}{2}\right)\right)^{2}+0.05\right)
$$

This equation is similar to the conditions encountered during optical photolithography when an optical beam exhibits self-interference in the resist upon encountering a reflective substrate. A small amount of curvature rate dependence was added for stability of the simulation equal to $\epsilon=\frac{\Delta x}{40}$. The etch time is 35.5 time units, $\frac{\Delta t}{\Delta x}=0.25$ and the contours were plotted every 3.55 time units. (Figures 8,9 and 10).

\subsection{Vertical Etching}

In this example, the etch rate function is:

$$
R(x, y, \theta)=e^{-0.32(x-5)^{2}} \cos \theta
$$

This equation does not exactly correspond to any particular process, but is useful in examining the behavior of simulators under anisotropic conditions. The characteristics of the surface all point directly downwards, 


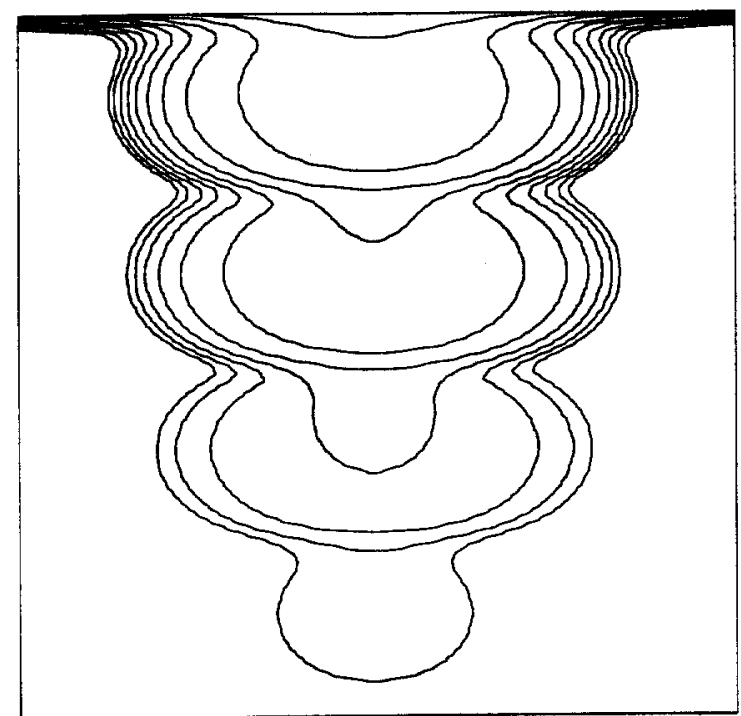

Figure 8: Photolithography 100x100

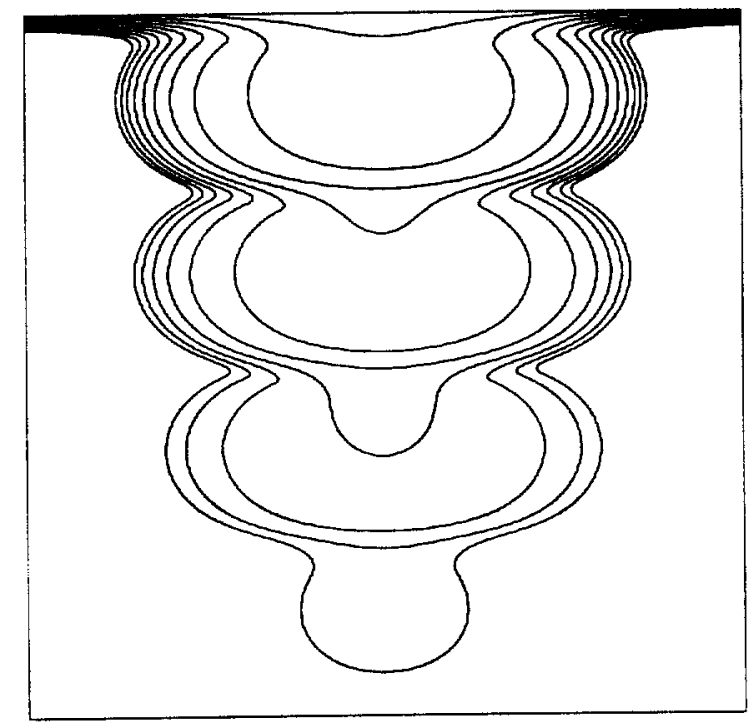

Figure 9: Photolithography 200x200 
thereby allowing easy detection of incorrectly determined characteristics. The etch time is 9.5 time units, $\frac{\Delta t}{\Delta x}=0.25$ and the contours were plotted every 0.25 time units. The added curvature was $\epsilon=\frac{\Delta x}{4}$. (Figures 11, 12 and 13).

\subsection{Ion Milling Applied to Uneven Topography}

In this example, the etch rate function is:

$$
\begin{aligned}
R(\theta)=\cos \theta & (1.454743 \cos \theta \\
& -0.464719 \cos 3 \theta \\
& +0.015573 \cos 5 \theta \\
& -0.005669 \cos 7 \theta \\
& -0.010000 \cos 9 \theta \\
& +0.010552 \cos 11 \theta \\
& -0.0006204 \cos 13 \theta \\
& +0.005725 \cos 15 \theta)
\end{aligned}
$$

The trench is formed by a rectangle extending from $x=3$ to $x=7$ and $y=0$ to $y=5$. This example is similar to an example found in [5]. The rate function is identical, although the size of the trench may be different, since it was not specificed in the original reference. This particular etch rate function describes a non-convex sputter yield curve. Because of the non-convexity of this function, the faceting that is exhibited at the upper corners and the rarefaction fan at the lower corners develop. These faceting behaviors are characteristic of non-convex functions, and are difficult to simulate for many approaches. The etch time is 4.5 time units, $\frac{\Delta t}{\Delta x}=0.25$ and the contours were plotted every 0.25 time units. The added curvature was $\epsilon=\frac{\Delta x}{4}$. (Figures 14, 15 and 16).

\subsection{Ion Milling with Uneven Ion Beams}

In this example, the etch rate function is:

$$
\begin{aligned}
& R(x, y, \theta)=e^{\frac{-(x-5)^{2}}{4}} \cos \theta \quad(1.454743 \cos \theta \\
& -0.464719 \cos 3 \theta \\
& +0.015573 \cos 5 \theta \\
& -0.005669 \cos 7 \theta
\end{aligned}
$$




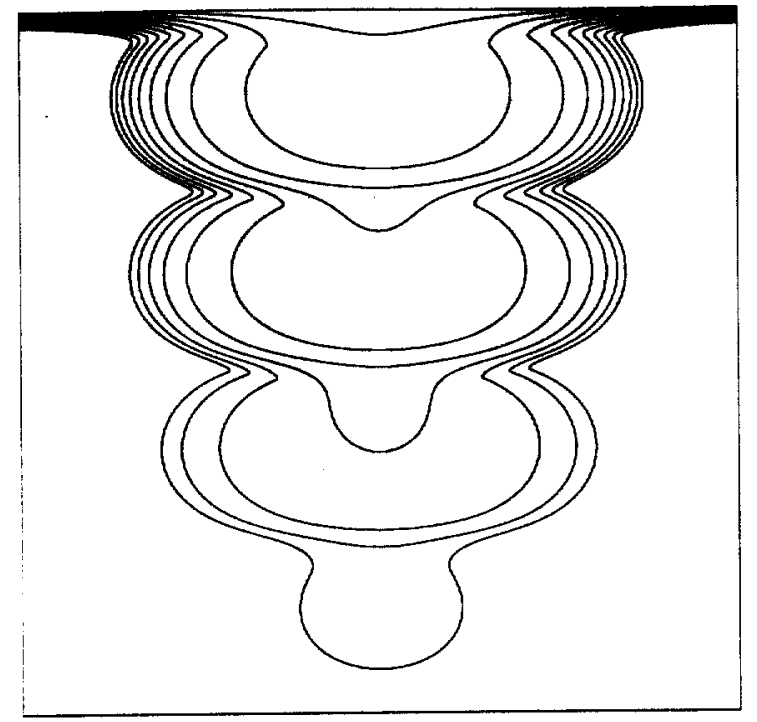

Figure 10: Photolithography 400x400

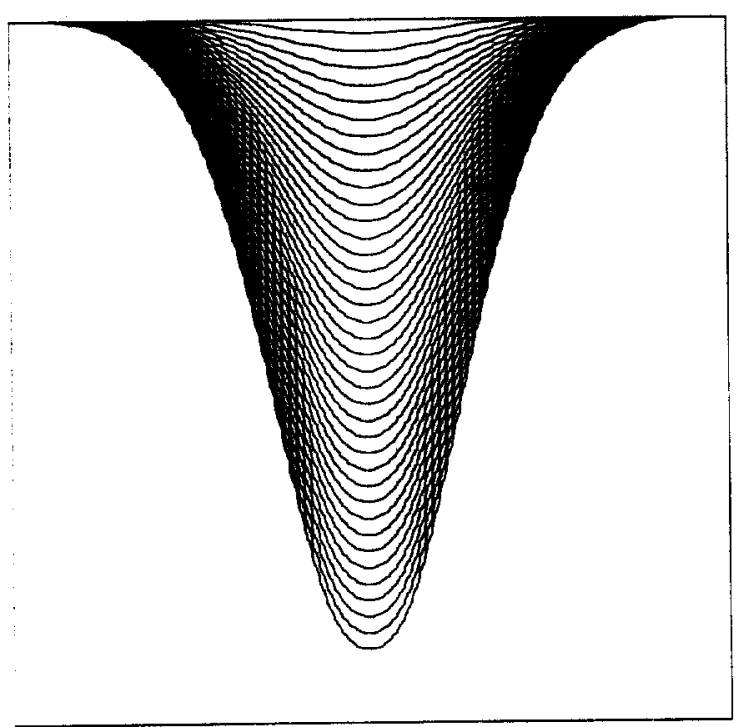

Figure 11: Exponential Vertical 100×100 


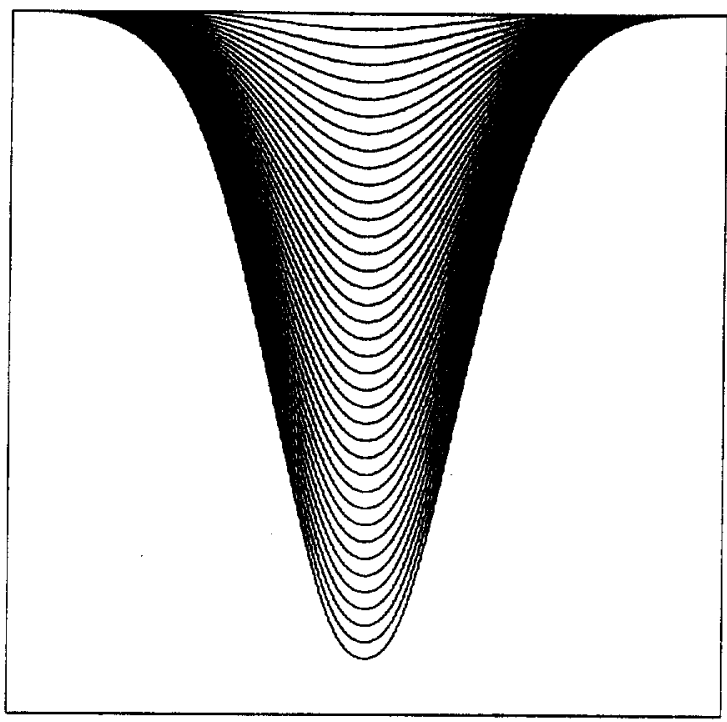

Figure 12: Exponential Vertical 200x200

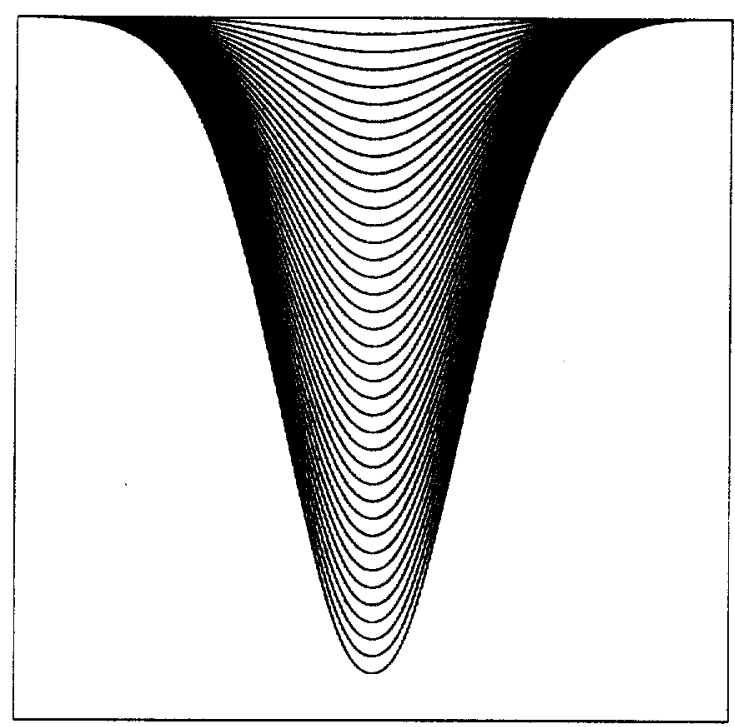

Figure 13: Exponential Vertical $400 \times 400$ 


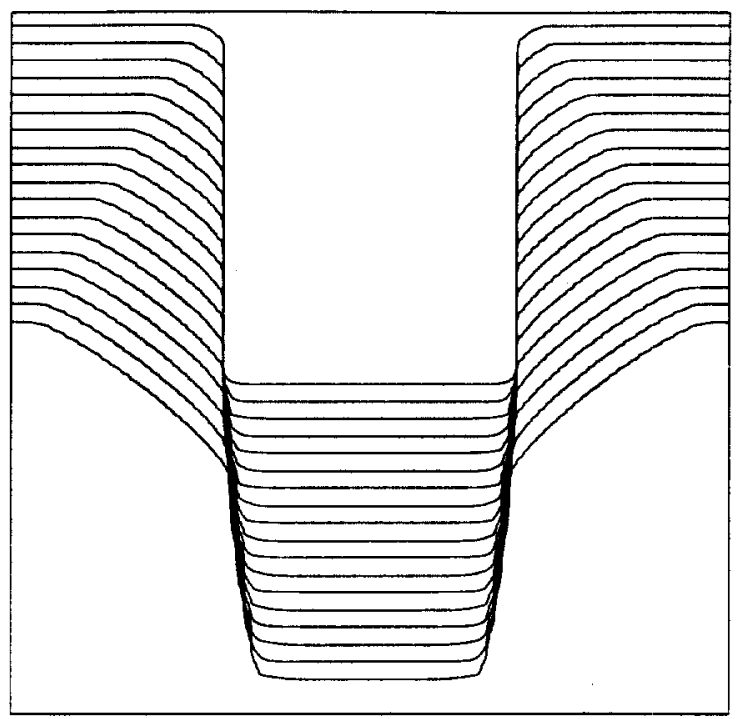

Figure 14: Ion Milled Trench 100x100

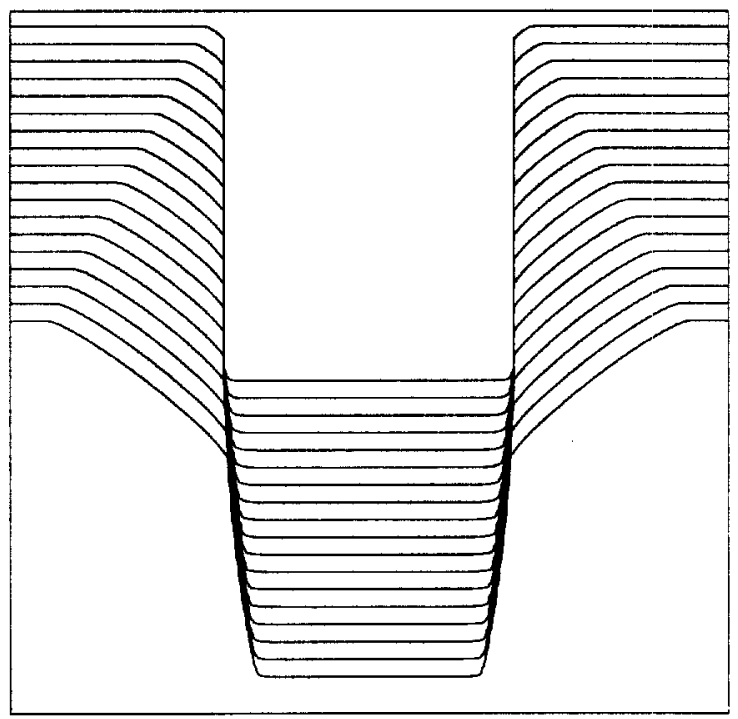

Figure 15: Ion Milled Trench 200x200 


$$
\begin{aligned}
& -0.010000 \cos 9 \theta \\
& +0.010552 \cos 11 \theta \\
& -0.0006204 \cos 13 \theta \\
& +0.005725 \cos 15 \theta)
\end{aligned}
$$

This example is identical to an example found in [5]. Non-convex and spatially varying etch rates are combined here, to give a simulation of ion milling of a surface using an exponentially varying ion beam. While the surface first shows a gaussian shape, as in the vertical etching example, the non-convex term gradually dominates. This is evidenced by the formation of the 'floor' of the etch profile. The ability to perform this example, shows that the simulator can generate accurate results for a wide variety of different types of etch rates. The etch time is 9.5 time units, $\frac{\Delta t}{\Delta x}=0.25$ and the contours were plotted every 0.25 time units. The added curvature was $\epsilon=$ $\frac{\Delta x}{4}$. (Figures 17, 18 and 19).

\section{Conclusions}

The Volume of Fluids method of representing boundaries between two fluids has been applied to the problem of surface evolution in two dimensions. The Volume of Fluids picture of surface evolution has been rigidly defined. A new, and significantly faster method of evaluating the orientation of the surface in each boundary cell has been developed and applied. A fast method of evaluating surface curvature has been presented and included as a diffusive term. Techniques for advancing the volume fractions have been shown that include the ability to evaluate edge centered divergence terms as well as cell centered divergence terms.

The Volume of Fluid surface advancement algorithm has been shown to be capable of accurately simulating several problems of interest to the semiconductor manufacturing community. The algorithm has also been shown to be accurate on problems whose etch rates are spatially varying and are non-convex with respect to surface orientation. The technique is also fully two-dimensional and able to handle complex undercutting.

To maintain a valid surface according to the entropy condition, it is necessary to add a diffusive term that is dependent on the curvature of the surface. This term is critical at strong shocks and at rarefaction fans whose bounding characteristics have velocities with opposite signs. In this implementation, a modifying effect to the etch rate that is proportional to 


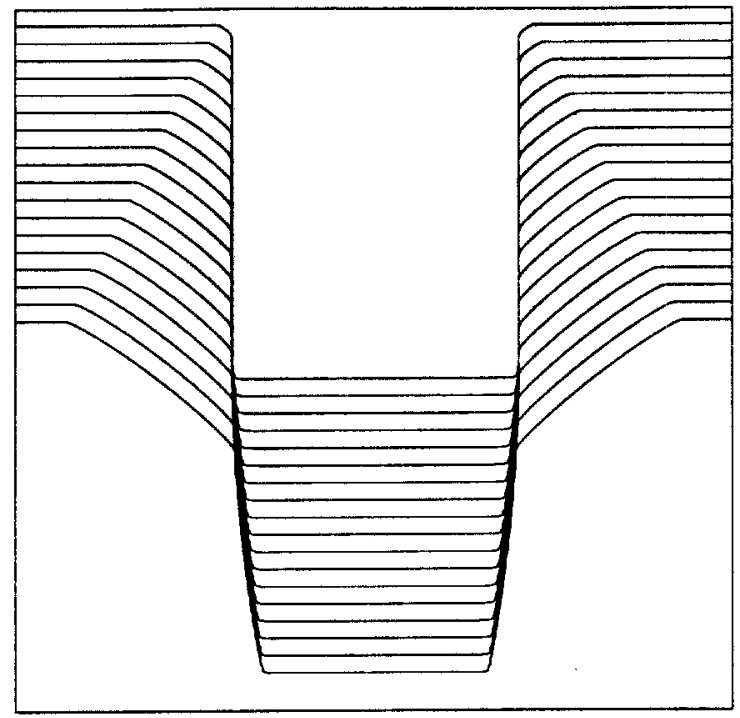

Figure 16: Ion Milled Trench 400x400

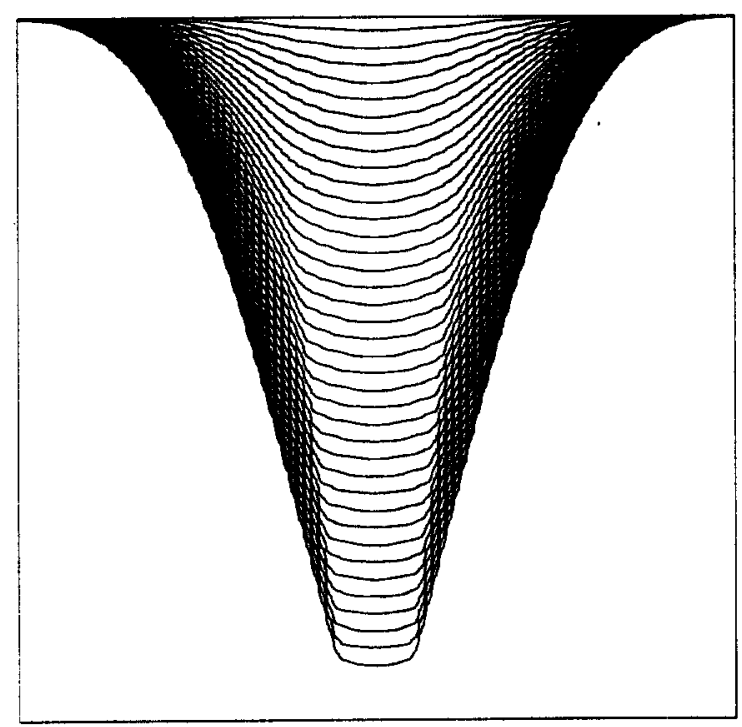

Figure 17: Exponential Ion Beam 100x100 


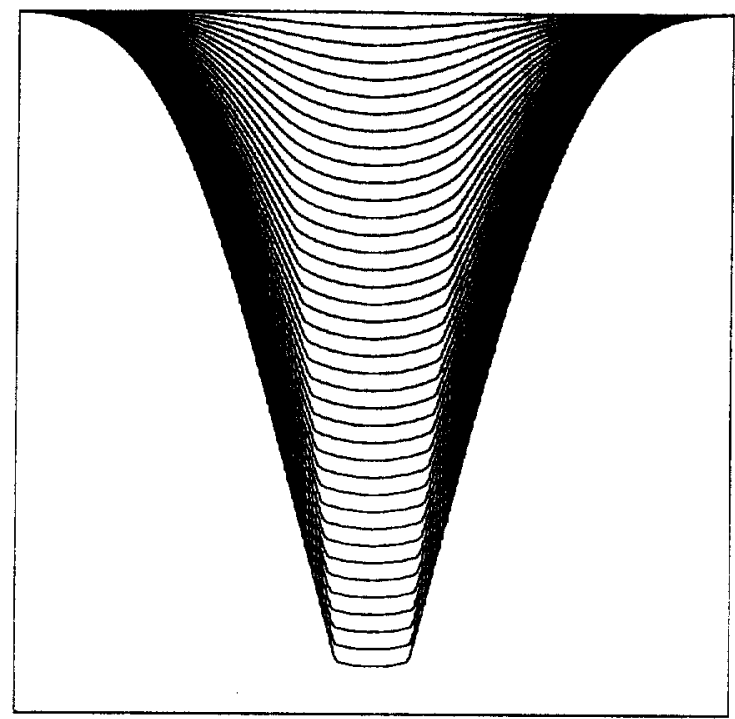

Figure 18: Exponential Ion Beam 200x200

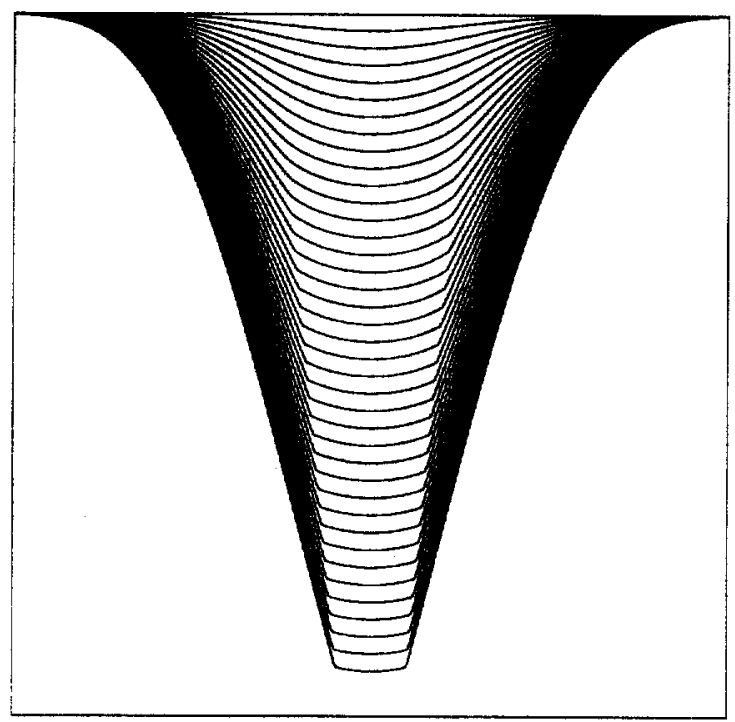

Figure 19: Exponential Ion Beam 400x400 
the curvature was added. Methods of selectively adding curvature to only these locations are being investigated.

The accuracy of the algorithm seems to be quite good, based on the similarity of the profiles for differing grid sizes. (The ripples in the surface in the low resolution plots are an artifact of the plotting program.) The amount of CPU resources consumed seems quite significant. The demand on the program to solve the Riemann problem at each cell edge contributes significant overhead, as opposed to methods used by other simulators [1][2] such as Lax-Freidrichs [7]. These methods, however, utilize significant amounts of numerical diffusion to maintain stability in the non-convex case, thereby requiring smaller grid sizes to improve accuracy. Improvements to the existing implementation of the software, in addition, will decrease the number of CPU cycles currently consumed. This approach, therefore, is expected to be competitive with other first order techniques, because it can operate accurately with coarser grids. In addition, it is also possible to remove the necessity of performing the Riemann solve in the VoF approach entirely by converting to a Lax-Freidrichs based advancement scheme.

Extension to three dimensions is straightforward. All the major components of the algorithm have clear three dimensional equivalents.

\section{Future Work}

Improvements in the code will be implemented to accelerate the rate of execution. Application of this technique to more complex problems in semiconductor manufacturing will be performed. The algorithm will be improved to handle etch rates that contain simultaneous etching and deposition terms and advancement rates that are determined by radiative transport of material. The Volume of Fluid surface advancement method will be extended to three dimensions. Comparison to higher order PDE schemes will be performed. Second order accuracy through the use of predictor-corrector methods can be achieved.

\section{References}

[1] Adalsteinsson, D. and Sethian, J.A., "A Level Set Approach to a Unified Model for Etching, Deposition, and Lithography I: Algorithms and Two Dimensional Simulations," Journal of Computational Physics, vol. 120, pp. 128-144, 1995. 
[2] Adalsteinsson, D. and Sethian, J.A., "A Level Set Approach to a Unified Model for Etching, Deposition, and Lithography II: Three Dimensional Simulations," Journal of Computational Physics, vol. 120, pp. 128-144, 1995.

[3] Dill, F.H., Hornberger, W.P., Hauge, P.S., and Shaw, J.M., "Characterization of Positive Photoresist," IEEE Transactions on Electron Devices, vol. ED-22, pp. 456-464, July 1975.

[4] Ferguson, R., Hutchinson, J.M., Spence, C.A., and Neureuther, A.R., "Modeling and Simulation of a Deep-UV Acid Hardening Resist," Journal of Vacuum Science and Technology B, vol. 8, pp. 1423-1427.

[5] Hamaguchi, S., Dalvie, M., Farouki, R.T., and Sethuraman, S., "A Shock-Tracking Algorithm for Surface Evolution Under Reactive-Ion Etching," Journal of Applied Physics, vol. 74, no. 8, Oct. 1993.

[6] Kim, D., Oldham, W., and Neureuther, A., "Development of Positive Photoresist," IEEE Transactions on Electron Devices, vol. 31. pp. 17301735, Dec. 1984.

[7] LeVeque, R.J., Numerical Methods for Conservation Laws, Birkhauser Verlag, 1990.

[8] Mack, C., "Development of Positive Photoresists," Journal of ElectroChemical Society, vol. 134, no. 1, pp.148-152, 1987.

[9] Osher, S. and Sethian, J., Journal of Computational Physics vol. 79, no. $12,1988$.

[10] Pilliod, J. and Puckett, E.G., "Second-Order Volume-of-Fluid Algorithms for Tracking Material Interfaces," Preprint.

[11] Puckett, E., "A Volume-of-Fluid Interface Tracking Algorithm with Applications to Computing Shock Wave Refraction," Proceedings of the 4th International Symposium on Computational Fluid Dynamics, pp.933-938, U.C. Davis, 1991.

[12] Sethian, J. and Strain, J., "Crystal Growth and Dendritic Solidification," Journal of Computational Physics, v. 98, pp. 231-253, Feb. 1992.

[13] Tam, N.N., "Resist Mechanisms and Models in Electron Beam Lithography," Ph.D. Dissertation, University of California, Berkeley, 1991. 
[14] Wolf, S. and Tauber, R.N., Silicon Processing in the VLSI Era, Volume 1: Process Technology, Lattice Press, 1986.

[15] Zel'dovich, Y.B. and Raizer, Y.P., Physics of Shock Waves and HighTemperature Hydrodynamic Phenomena, Academic Press, 1966. 


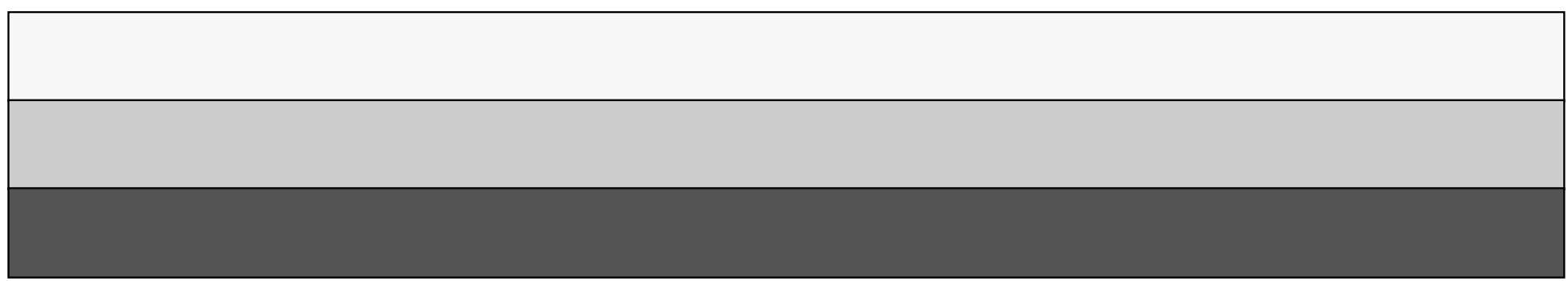

